\title{
INDICADORES E ÍNDICES DE CALIDAD DE LOS SUELOS (ICS) CEBADEROS DEL SUR DEL ESTADO DE HIDALGO, MÉXICO ${ }^{1}$
}

\author{
Judith Prieto-Méndez ${ }^{2}$, Francisco Prieto-García ${ }^{3}$,Otílio Arturo Acevedo-Sandoval ${ }^{2}$, \\ María Aurora Méndez-Marzo ${ }^{3}$
}

\section{RESUMEN}

Indicadores e índices de calidad de los suelos (ICS) cebaderos del sur del estado de Hidalgo, México. El objetivo de este trabajo fue establecer indicadores e índices de calidad para suelos (ICS), que permitan realizar evaluaciones y estimaciones rápidas en suelos cebaderos del sur del estado de Hidalgo, México. Ocho indicadores fueron normalizados en una escala 0-1 que representan, respectivamente, la peor y mejor condición desde el punto de vista de la calidad, independientemente de los valores absolutos medidos para cada indicador. Los máximos y mínimos para algunos atributos fueron establecidos para las condiciones óptimas. El ICS encontrado en esta región fue de 0,48 lo cual lo ubica en clase de moderada calidad. Este valor estuvo fuertemente influenciado por bajos valores de carbono orgánico del suelo (COS), como propiedad que más los está afectando. Esta disminución del COS resulta también la causa principal de los valores bajos de estabilidad de agregados e infiltración y un indicador de los valores de densidad aparente. Estos cambios en las propiedades físicas afectan la condición superficial del suelo provocando un incremento de los procesos de erosión, con la consiguiente pérdida de espesor del horizonte superficial, reflejada por el indicador correspondiente.

\section{ABSTRACT}

Soil quality (ISQ) indicators and indices of feedlots in the southern state of Hidalgo, Mexico. The objective of this study was to establish indicators and soil quality index (SCI) to enable rapid assessments and estimates in feedlot soils of the southern state of Hidalgo, Mexico. Consideration was given eight indicators were standardized on a 0-1 scale, representing respectively the worst and best condition from the standpoint of quality, regardless of the absolute values measured for each indicator. The maximum and minimum values for some attributes were set to optimal conditions. The SCI found in this region was 0.48 , which places it as moderate quality. This value was influenced by low values of soil organic carbon (SOC), as property that is affecting these soils. This decrease of SOC is also the main cause of the low values of aggregate stability and infiltration and an indicator of the values of bulk density. These changes affect the physical properties of soil surface condition leading to increased erosion processes, with consequent loss of thickness of the surface horizon, reflected by the indicator.

Key words: quality indicators, organic carbon, quick estimates, aggregate stability.

Palabras clave: indicadores de calidad, carbono orgánico, estimaciones rápidas, estabilidad de agregados.

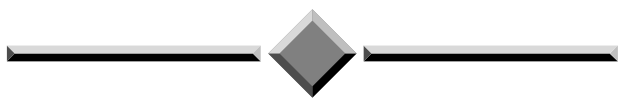

1 Recibido: 4 de junio 2012. Aceptado: 1 de abril, 2013. Este trabajo es parte de la tesis doctoral del primer autor, dentro del Programa Educativo del Doctorado en Ciencias Ambientales, Universidad Autónoma del estado de Hidalgo, México.

2 Universidad Autónoma del Estado de Hidalgo. Instituto de Ciencias Agropecuaria. Rancho Universitario. Ciudad de Tulancingo. Hidalgo. México.jud_292003@yahoo.com.mx (Autor para correspondencia),oacevedo@uaeh.edu.mx

3 Universidad Autónoma del Estado de Hidalgo. Instituto de Ciencias Básicas e Ingeniería. Ciudad Universitaria. Carretera PachucaTulancingo, Km 4.5. C.P. 42076.Pachuca. Hidalgo. México.prietog@uaeh.edu.mx, jumafran@yahoo.es

(C) 2013 Agronomía Mesoamericana es desarrollada en la Universidad de Costa Rica y se encuentra licenciada con Creative Commons Reconocimiento-NoComercial-SinObraDerivada 3.0 Costa Rica. Para más información escríbanos a pccmca@ucr.ac.cr 


\section{INTRODUCCIÓN}

La Conferencia de las Naciones Unidades sobre el Ambiente y el Desarrollo Río '92, marcó un hito muy especial al establecer la necesidad de desarrollar y aplicar diferentes metodologías para determinar el estado del ambiente y monitorear los cambios ocurridos a nivel local, nacional, regional y global (Naciones Unidas 1993). La determinación de estos cambios podría ayudar a realizar una mejor evaluación de los diferentes problemas ambientales, identificar y evaluar los resultados de la aplicación de las convenciones internacionales y los programas de acción, asî como también, orientar las políticas nacionales. Una amplia gama de metodologías se están utilizando para evaluar el impacto de la actividad agropecuaria sobre los recursos naturales y en especial, el cambio de uso y manejo de suelos (Archer et al. 2002, Breuer et al. 2006, Hati et al. 2007, Cantú et al. 2009).

El desarrollo de diversas metodologías ha determinado el uso generalizado de indicadores e índices para la evaluación de la calidad ambiental, calidad de suelos, sustentabilidad, desarrollo sustentable, riesgo, vulnerabilidad, planificación territorial, entre otros. El antecedente más importante surgió de la Organisation for Economic Cooperation and Development (OECD 1991) cuando publicó un set preliminar de indicadores ambientales. Posteriormente, otras organizaciones han desarrollado programas donde se establecieron listas de indicadores para evaluar la calidad ambiental (Cantú et al. 2009), tales como, FAO, Banco Mundial, y los programas de Naciones Unidas para el desarrollo y para el medio ambiente (UNDP y UNEP, por sus siglas en Inglés, respectivamente).

El concepto de sustentabilidad y resiliencia del suelo fue descrito por Blum y Santelises (1994) basado en seis funciones ecológicas y humanas: suelo como productor de biomasa; como reactor con filtros, como buffer, transformador de materia para proteger el ambiente, el agua subterránea y la cadena de alimentos de la contaminación, como hábitat biológico y reserva genética, como medio físico y como fuente de recursos y de herencia cultural. Estos conceptos y los sugeridos por Warketin (1996) fueron las bases a partir de las cuales la Soil Sciencie Society of America estableció el concepto de calidad del suelo (Karlen et al. 1997). A partir de estos conceptos se establecieron indicadores cuantitativos de calidad del suelo (Doran y Parkin 1994, 1996, Doran et al. 1996).
Un indicador es una variable que simplifica información relevante haciendo que una condición de interés se haga perceptible y que cuantifica, mide y comunica, en forma comprensible, información relevante. Deben ser preferiblemente variables cuantitativas, aunque pueden ser cualitativas, nominales, de rango u ordinales, especialmente cuando no hay disponibilidad de información cuantitativa, el atributo no es cuantificable o cuando los costos para cuantificar son demasiado elevados (Volveré y Amézquita 2009). Las principales funciones son: evaluar condiciones o tendencias, comparar transversalmente sitios o situaciones, para evaluar metas y objetivos, proveer información preventiva temprana y anticipar condiciones y tendencias futuras.

Los indicadores deben ser limitados en número, manejables por diversos tipos de usuarios, sencillos, fáciles de medir y tener un alto grado de agregación, es decir, deben ser propiedades que resuman otras cualidades o propiedades; interdisciplinarios; en lo posible deberán contemplar la mayor diversidad de situaciones; por lo tanto, incluir todo tipo de propiedades de los suelos (químicas, físicas, biológicas, etc.), tener una variación en el tiempo tal que sea posible realizar un seguimiento de las mismas; asimismo, no deberán poseer una sensibilidad alta a los cambios climáticos y/o ambientales pero la suficiente como para detectar los cambios producidos por el uso y manejo de los recursos (Gallopin 1997, Doran y Parkin 1996, Doran y Zeiss 2000, Volveré y Amézquita 2009).

A partir de la experiencia del Banco Mundial, Segnestam (2002) señaló la importancia de establecer: la línea de base o de inicio de una actividad que puede impactar positiva o negativamente sobre el ambiente, umbrales para controlar o hacer el seguimiento de impactos negativos que no deben exceder un predeterminado umbral y además objetivos o metas que permitan evaluar si el impacto positivo de una respuesta es suficientemente largo.

Se han desarrollado listas de indicadores de uso universal pensando en todas las situaciones posibles y todos los suelos posibles (Doran y Parkin 1994, 1996). Por otra parte, se han presentado listas pensadas para situaciones regionales o locales (Brejda et al. 2000, Segnestam 2002, Cantú et al. 2002, Lilburne et al. 2004). El objetivo de este trabajo fue establecer indicadores e índices de calidad para suelos (ICS), que permitan realizar evaluaciones y estimaciones rápidas en suelos cebaderos del sur del estado de Hidalgo, México. 


\section{MATERIALES Y MÉTODOS}

El área de estudio comprende un grupo de parcelas localizadas en los municipios de Apan, Almoloya y Emiliano Zapata, todas ellas con suelos dedicados al cultivo de cebada calidad maltera, como sistema de monocultivo por más de 40 años, ubicados en el sur del Estado de Hidalgo, México.

Se propuso, siguiendo los criterios establecidos (Breuer et al.2006, Hati et al. 2007, Cantú et al. 2009) aplicar como indicadores para evaluaciones rápidas de calidad de los suelos cebaderos los siguientes parámetros:

- $\mathrm{pH}$

- Carbono orgánico (CO5)

- Porcentaje de saturación de bases

- Porcentaje de agregados estables en agua (>0,5 $\mathrm{mm})$.

- Velocidad de infiltración

- Densidad aparente

- Potencial Zeta pZ

- Espesor del Horizonte A

Otro trabajo publicado (Rodríguez et al. 2009) refiere la inclusión de otros parámetros como la respiración de los suelos y los contenidos de fósforos. En el presente estudio se tuvo en consideración la inclusión del potencial Zeta (pZ), no reportado en las bibliografías consultadas. Este estudio se realizó entre febrero 2009 y mayo 2011.

La determinación del $\mathrm{pH}$ de los suelos, se realizó por potenciometría (relación suelo-agua 1:2), según lo establece la NOM 021 RECNAT (2000) y partiendo del extracto de saturación (metodología AS-16). El contenido de carbono orgánico se determinó por el método de Walkley y Black (Jackson 1987). La saturación de bases se determinó por el método del acetato de amonio (NOM-021-RECNAT-2000; metodología AS-21). El porcentaje de agregados estables en agua $(>0,5 \mathrm{~mm}$ ) se obtuvo de acuerdo a Certini et al. (2002) y atendiendo a lo establecido en USDA-NRCS (1999). La velocidad de infiltración se realizó con el método del doble anillo (ASTM 1993, USDA-NRCS 1999) y la densidad aparente se determinó por el método del cilindro (Blake et Hartge 1986, USDA-NRCS 1999). El espesor del horizonte A de los suelos muestreados fue valorado en campo (Soil Survey Staff 2006).

La metodología de indicadores tiene como condición esencial, apoyarse en una base cartográfica para que a partir de ella sea factible el análisis en el espacio y en el tiempo. En este caso la cartografía de base se realizó utilizando mapas satelitales (Figura 1).

Para realizar la evaluación de la calidad de los suelos cebaderos, teniendo en cuenta el número de indicadores mínimos que se sugieren, se han propuesto

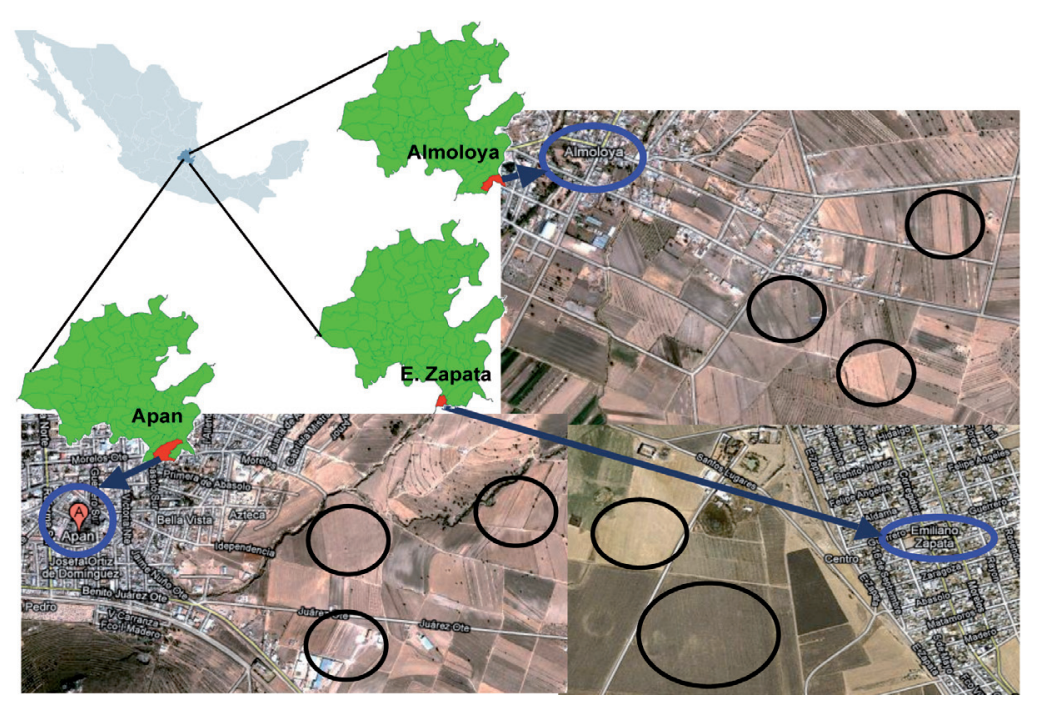

Figura 1. Ubicación de las parcelas estudiadas de suelos cebaderos en los municipios de Almoloya, Apan y Emiliano Zapata. Estado de Hidalgo, México. 2009. 
los indicadores e índices que se observan en el Cuadro 1. Para la obtención de un valor único de cada parámetro por cada municipio, se realizó un promedio ponderado de acuerdo a la proporción que representa cada manejo en el área total estudiada. Luego los indicadores fueron normalizados utilizando una escala 0-1 que representan, la peor y mejor condición desde el punto de vista de la calidad, independientemente de los valores absolutos medidos para cada indicador y obtenidos como resultados experimentales.

Los valores máximos y mínimos fueron establecidos de diferentes formas para cada indicador. Para algunos atributos, especialmente para las condiciones óptimas, se tuvieron en cuenta umbrales calculados a partir de los valores de los suelos reportados (Breuer et al. 2006, Hati et al. 2007, Cantú et al. 2007, Cantú et al. 2009), mientras que en otros se utilizaron criterios teóricos reportados para suelos dedicados al cultivo de cebada (Vera et al. 2002, Álvarez et al. 2006).

Para el COS, se consideró como mínimo, el requerimiento para cumplir con la condición de mólico (Soil Survey Staff 2006) y como máximo el promedio de los valores medidos en los suelos de referencia (Álvarez et al. 2006, Hati et al. 2007, Cantú et al. 2007, Cantú et al. 2009); se considera el mínimo en $1 \%$ y el máximo en $7 \%$, lo cual obedece igualmente a la clasificación que indica Boulding (1995) para suelos no volcánicos. El valor mínimo de $\mathrm{pH}$ fue establecido considerando el punto de toxicidad para el desarrollo del cultivo de cebada en la zona (pH de 5,0) y el máximo de calidad correspondió al pH que en mayores cuantías puede considerarse como apto $(8,5)$ para el cultivo de cebada (Vera et al. 2002, Álvarez et al. 2006, Soil Survey Staff 2006, Pulido et al. 2009). Para la saturación de bases se tomaron el valor mínimo (50\%) y el máximo (100\%) requeridos para cumplir con la condición de mólico antes mencionada (Soil Survey Staff 2006). Para el indicador de agregados estables en agua, el valor máximo se obtuvo promediando valores medidos reportados en diversos trabajos (Shepherd et al. 2001, Holeplass et al. 2004, Lado et al. 2004, Márquez et al. 2004, Bronick y Lal 2005, Pulido et al. 2009). El mínimo correspondió a los menores valores medidos en la región.

En el caso de la velocidad de infiltración, se tomó como mínimo la velocidad a la cual se han documentado problemas de infiltración en la región (Gómez y Magallanes 2004) y como máximo los valores de infiltración esperados de acuerdo a las características del suelo (Soil Survey Staff 2006) que en este caso clasifican entre franco arcillo arenosos y franco arenosos. La densidad aparente mínima corresponde al promedio de los valores medidos en los suelos de referencia (Márquez et al. 2004, Bronik y Lal 2005, Pulido et al. 2009) y la máxima a los valores máximos medidos en la región.

Para el indicador de potencial zeta (pZ), es la primera vez que se utiliza para tales fines, por lo que se tomó como máximo y mínimo, <-30 $\mathrm{mV}$ y >+30 $\mathrm{mV}$, valores sobre los que se ha reportado que resultan estables las suspensiones coloidales de los extractos de saturación de suelos (Prieto et al. 2009).

Cuadro 1. Indicadores propuestos para evaluación de calidad de suelos (ICS) cebaderos, unidades de medida, e índices como valores máximos y mínimos definidos para los Municipios. Estado de Hidalgo, México. 2011.

\begin{tabular}{lccc}
\hline Indicador & U.M. & \multicolumn{2}{c}{ ICS } \\
\cline { 3 - 4 } & & Máx. & Mín. \\
\hline $\mathrm{pH}$ & - & 8,50 & 5,00 \\
$\mathrm{C}_{\text {orgánico }}(\mathrm{COS})$ & $\%$ & 6,00 & 1,00 \\
Saturación de Bases & $\%$ & 100 & 50 \\
Agregados estables en agua & $\%$ & 75 & 10 \\
Velocidad de infiltración & $\mathrm{cm} / \mathrm{h}$ & 10 & 1 \\
Densidad aparente & $\mathrm{Mg} / \mathrm{m}^{3}$ & 1,50 & 1,05 \\
Potencial Zeta (pZ) & $\mathrm{mV}$ & +30 & -30 \\
Espesor de horizonte A & $\mathrm{cm}$ & 45 & 0 \\
\hline
\end{tabular}

U.M.: Unidad de medida. ICS: Indicadores de calidad de suelos. 
En el caso del indicador espesor del horizonte A, el máximo correspondió al medido en promedio en suelos de referencia $(45 \mathrm{~cm})$, mientras que el mínimo se estableció como cero (nivel superficial de la capa arable). Finalmente, se estableció un índice de calidad de suelos (ICS) promediando los valores de todos los indicadores. Para la interpretación del ICS se utilizó una escala de transformación en cinco clases de calidad de suelo de 1 a 5 (Cuadro 2) y según propuesta que reportan Cantú et al. (2009).

Cuadro 2. Clases de calidad de suelos. Estado de Hidalgo, México. Cantú et al. 2009.

\begin{tabular}{lcc}
\hline $\begin{array}{l}\text { Índice de calidad } \\
\text { de suelos }\end{array}$ & Escala & Clase \\
\hline Muy alta calidad & $0,80-1,00$ & 1 \\
Alta calidad & $0,60-0,79$ & 2 \\
Moderada calidad & $0,40-0,59$ & 3 \\
Baja calidad & $0,20-0,39$ & 4 \\
Muy baja calidad & $0,00-0,19$ & 5 \\
\hline
\end{tabular}

Existen dos situaciones posibles (Cantú et al. 2007, 2009): la primera cuando el valor máximo del indicador $\left(\mathrm{I}_{\text {máx }}\right)$ corresponde a la mejor situación de calidad de suelo (Valor normalizado del indicador: $\mathrm{V}_{\mathrm{n}}$ = 1) y el cálculo es:

$$
\mathrm{V}_{\mathrm{n}}=\left(\mathrm{I}_{\mathrm{m}}-\mathrm{I}_{\text {min }}\right) /\left(\mathrm{I}_{\text {max }}-\mathrm{I}_{\text {min }}\right)
$$

La otra situación es cuando el valor $\mathrm{I}_{\max }$ corresponde a la peor situación de calidad de suelo $\left(\mathrm{V}_{\mathrm{n}}=0\right)$ y se calcula como:

$$
\mathrm{V}_{\mathrm{n}}=1-\left(\mathrm{I}_{\mathrm{m}}-\mathrm{I}_{\min } / \mathrm{I}_{\max }-\mathrm{I}_{\text {min }}\right)
$$

donde $\mathrm{V}_{\mathrm{n}}=$ valor normalizado, $\mathrm{I}_{\mathrm{m}}=$ medida del indicador, $\mathrm{I}_{\max }=$ valor máximo del indicador, $\mathrm{I}_{\min }=$ valor mínimo del indicador.

\section{RESULTADOS Y DISCUSIÓN}

De la evaluación de la calidad de los suelos cebaderos del sur del estado de Hidalgo, teniendo en cuenta el número de indicadores mínimos que se sugieren, y los valores únicos normalizados obtenidos para cada municipio; el indicador que presentó el menor valor en promedio para los tres municipios, fue el COS $(0,18)$ mientras que el mayor correspondió a la saturación de bases $(0,94)$. El pH y la densidad aparente presentaron valores promedios intermedios $(0,49-0,59)$, cercanos al $50 \%$ y los restantes indicadores oscilaron entre valores desde 0,34 hasta 0,45 . El nuevo indicador que se propone, el pZ, marcó un ICS promedio de la región sur de 0,71 (Cuadro 3).

$\mathrm{El}$ indicador $\mathrm{pH}$ presenta en promedio un valor de calidad de 0,55. En la mayoría de los suelos de la

Cuadro 3. Indicadores e índices de calidad de suelos (ICS) por municipios. Estado de Hidalgo, México. 2011.

\begin{tabular}{lcccc}
\hline Indicador & \multicolumn{3}{c}{ Valores de ICS por indicadores } & ICS \\
\cline { 2 - 5 } & Apan & Almoloya & E. Zapata & Promedio \\
\hline $\mathrm{pH}$ & $0,59 \mathrm{a}$ & $0,49 \mathrm{~b}$ & $0,56 \mathrm{a}$ & $\mathbf{0 , 5 5}$ \\
$\mathrm{C}_{\text {organico }}$ & $0,20 \mathrm{c}$ & $0,18 \mathrm{c}$ & $0,17 \mathrm{c}$ & $\mathbf{0 , 1 8}$ \\
Saturación de Bases & $0,94 \mathrm{~d}$ & $0,89 \mathrm{e}$ & $0,98 \mathrm{~d}$ & $\mathbf{0 , 9 4}$ \\
Agregados estables en agua & $0,41 \mathrm{f}$ & $0,38 \mathrm{f}$ & $0,23 \mathrm{~g}$ & $\mathbf{0 , 3 4}$ \\
Velocidad de infiltración & $0,56 \mathrm{~h}$ & $0,42 \mathrm{i}$ & $0,38 \mathrm{j}$ & $\mathbf{0 , 4 5}$ \\
Densidad aparente & $0,56 \mathrm{k}$ & $0,52 \mathrm{k}$ & 0,48 & $\mathbf{0 , 5 2}$ \\
Potencial Zeta (pZ) & $0,67 \mathrm{~m}$ & $0,83 \mathrm{n}$ & $0,62 \mathrm{~m}$ & $\mathbf{0 , 7 1}$ \\
Espesor de horizonte A & $0,36 \mathrm{o}$ & $0,34 \mathrm{o}$ & $0,32 \mathrm{o}$ & $\mathbf{0 , 3 4}$ \\
Valores promedios & $\mathbf{0 , 5 1} \mathbf{p}$ & $\mathbf{0 , 5 1} \mathbf{~ p}$ & $\mathbf{0 , 4 9} \mathbf{~ p}$ & $\mathbf{0 , 5 0}$ \\
\hline
\end{tabular}

Letras diferentes en filas, representan diferencias significativas $(\mathrm{p}<0,05)$. 
región existe una tendencia a la disminución del $\mathrm{pH}$ en el horizonte superficial (A) respecto a los suelos de referencia. Esta situación también ha sido reportada por otros investigadores (Pulido et al. 2009), cuando se refieren a que en sistemas de monocultivos esta tendencia se observa para muchos tipos de suelos. Sin embargo, los valores medidos aún distan considerablemente del punto de toxicidad establecido para el cultivo de cebada $(<5,0)$.

El valor promedio del indicador COS para los suelos cebaderos de la región sur del estado de Hidalgo, evidencia una disminución en la calidad de un $82 \%$ respecto de los suelos tomados como referencia. $\mathrm{La}$ marcada disminución de la materia orgánica ha sido observada en diversas investigaciones en la región (López et al. 2005, Prieto et al. 2009).

El valor del indicador saturación de bases es muy cercano al máximo de calidad $(0,94)$. En este caso también el indicador refleja en gran medida la situación de los suelos locales. Los materiales loésicos sobre los que se desarrollan estos suelos (Medina et al. 2006) son ricos en calcio y por lo tanto, la saturación de bases es muy alta, a pesar del uso de estos en sistema de monocultivo que data de más de 40 años y los bajos contenidos en materia orgánica.

El indicador de agregados estables en agua presenta un valor de calidad bajo, de 0,34. La importante disminución del porcentaje de macroagregados en la región, respecto de los suelos de referencia, refleja la influencia negativa del manejo en esta propiedad como fuera señalado por algunos autores a nivel local (López et al. 2005, Prieto et al. 2009) e internacional (Gregorich et al. 1994, Doran y Safley 1997, Cantú et al. 2007). El uso de sistemas de monocultivo corroboran las afectaciones en la textura de los suelos y con ello a la formación de agregados estables (Pulido et al. 2009).

El valor de 0,45 del indicador velocidad de infiltración señala que esta propiedad en la región evaluada, dista en un 55\% de los valores de infiltración esperados de acuerdo a las características del suelo (Soil Survey Staff 2006). Este parámetro se ve afectado en gran medida por los valores de los suelos del Municipio de Emiliano Zapata $(0,38)$, debido a que los suelos son clasificados como arenosos. En investigaciones realizadas, se ha señalado que la disminución de la velocidad de infiltración estaría asociada a la compactación del horizonte superficial y subsuperficial (Prieto et al. 2009). Se ha reportado (Unzueta et al. 2007) que el incremento de la capacidad de infiltración del suelo frente al agua (generalmente por efecto temporal) así como el aumento en la estabilidad de los agregados frente al agua y a los agentes mecánicos, pueden estar regidas por una acción como la disminución del potencial Zeta (pZ) de las partículas del suelo. Con la variación de acidez el pZ disminuye su negatividad y en algunos casos, puede alcanzar valores positivos. Estos cambios afectan la velocidad del flujo electroosmótico, ya que se ha observado que la tasa de flujo decrece cuando el pH del electrolito se acerca a la neutralidad o se incrementa la alcalinidad (De la Rosa et al. 2007).

El indicador densidad aparente reveló que los suelos tienen un nivel medio de compactación, dado que el valor promedio ponderado de la región se encuentra entre los valores mínimos de los sitios de referencia y los máximos medidos en la región. Este valor no sería restrictivo para el crecimiento de raíces del cultivo de cebada en este tipo de suelos (Vepraskas 1994, USDANRCS 1999).

En cuanto al pZ, que se refiere a la estabilidad de suspensiones coloidales en extracto de saturación de suelos, debe señalarse que es la primera vez que se reportan estos resultados. Se puede apreciar que en promedio se alcanza un ICS que los permite clasificar como suelos de alta calidad; sin embargo debe recordarse que estas suspensiones coloidales son de bajas concentraciones, por cuanto corresponden a bajos contenidos de materia orgánica (COS). Cuanto mayor sea esta fuerza dispersora (pZ), mayor estabilidad tendrá la solución y mayor será su capacidad para acarrear partículas en suspensión; o capacidad de carga (De la Rosa et al. 2007). En este sentido se puede entender que los extractos de saturación de los suelos se presentan con valores de $\mathrm{pZ}$ que los ubica en un umbral de leve dispersión a moderada, por poca concentración de materia orgánica disponible.

El valor bajo del indicador espesor del horizonte A $(0,34)$ muestra la marcada disminución respecto de los suelos de referencias (Holeplass et al. 2004, Lado et al. 2004, Márquez et al. 2004, Bronik y Lal 2005), que alcanza un $66 \%$ en estos suelos evaluados. Esto se debe principalmente a procesos de erosión hídrica que han sido estimados en la zona por mediciones directas en campo y por resultados de texturas franco arenosas.

El índice de calidad promedio de los suelos de esta región cebadera (ICS: 0,50) obtenido mediante 
este set mínimo de indicadores, se ubica en la clase 3, de moderada calidad de suelos (Cuadro 2). Este valor del ICS está fuertemente influenciado por el indicador COS, que es la propiedad más afectada por el manejo de estos suelos (sistema de monocultivo). El COS es considerado un atributo clave dada su marcada influencia sobre la mayoría de las propiedades del suelo (Gregorich et al. 1994). La disminución del COS sería la causa principal de los bajos valores del indicador estabilidad de agregados e infiltración y medio del indicador densidad aparente. Estos cambios en las propiedades físicas afectan la condición superficial del suelo provocando un incremento de los procesos de erosión, con la consiguiente pérdida de espesor del horizonte superficial, reflejada por el indicador correspondiente.

El set de indicadores utilizados para evaluar la calidad del suelo cumple con los criterios más importantes requeridos para su uso como indicadores. Se trata de un número mínimo de variables o atributos del suelo que integran información de otras asociadas, incorpora indicadores físicos, químicos y fisicoquímicos, que en su mayoría son de fácil medición. Los indicadores seleccionados reflejan, en términos de calidad, los cambios en cada una de las propiedades. En la construcción del set se consideraron las principales propiedades de los suelos del área para que los indicadores representen las condiciones locales.

$\mathrm{Se}$ descartaron indicadores que, si bien forman parte de listas muy usadas en otras partes del mundo (Doran y Safley 1997), no tienen validez local. Por ello, es importante señalar que estos indicadores de estado del recurso suelo, no son universales sino que deben ser elegidos en función del tipo de ambiente y suelo de la región en estudio. Estos resultados representan una visión del momento, para la situación de estos suelos en el período 2007 - 2010. Para darle el sentido temporal será necesario realizar mediciones secuenciales en lapsos de tiempo tales que permitan registrar cambios en los atributos utilizados vinculados a las condiciones de uso y manejo de los suelos (Cantú et al. 2007).

\section{AGRADECIMIENTOS}

Al Consejo Nacional de Ciencia y Tecnología (CONACYT) de México por el apoyo de la beca N 216385 durante la realización de los estudios doctorales.

\section{LITERATURA CITADA}

Álvarez, PA; Luna, M; Hernández, J; Lara, A; Salas, MA; Cabañas, B. 2006. Production systems of malting barley (Hordeum vulgare L.) in Zacatecas state, Mexico. Agric. Téc. Méx. 32(2):181-190.

Archer, N; Hess, T; Quinton, J. 2002. The water balance of two semiarid shrubs on abandoned land in SouthEastern Spain after cold season rainfall. Hydrology and Earth System Sciences 6(5):913-926.

ASTM (American Society for Testing and Materials). 1993. Annual book of standards. ASTM D 3385 - 88. Section 4 Construction. Volume 04.08: Soil and Rock, Dimension Stone; Geosynthetics. p. 452-458.

Blake, GR; Hartge, HK. 1986. Bulk density. In Klute, A. ed. Methods of soil analysis. Part 1. Agronomy Monograph $\mathrm{N}^{\mathrm{o}}$ 9. Am. Soc. Agron. Madison, Wisconsin, EE.UU. p. 363-375.

Blum, W; Santelises, AA. 1994. A concept of sustainability and resilience based on soil functions. In Greenland, DJ; Szboles, I. eds. Soil resilience and sustainable land use. CAB Int., Wallingford, Oxon, UK. p. 535-542.

Boulding, JR. 1995. Description and sampling of contaminated soils. A field guide, 2 ed. Lewis Publishers; Chapter 3. Boca Raton, FL, USA. 240 p.

Brejda, JJ; Moorman, B; Karlen, DL; Dao, T H. 2000. Identification of regional soil quality factors and indicators: I. Central and Southern High Plains. Soil Sci. Soc. Am. J. 64:2115-2124.

Breuer, L; Huisman, JA; Keller, T; Frede, HG. 2006. Impact of a conversion from cropland to grassland on $\mathrm{C}$ and $\mathrm{N}$ storage and related soil properties: Analysis of a 60 year chronosequence. Geoderma 133:6-18.

Bronick, CJ; Lal, R. 2005. Soil structure and management: a review. Geoderma 124:3-22.

Cantú, MP; Becker, AR; Bedano, JC; Musso, TB; Schiavo, HF. 2002. Evaluación de la calidad ambiental y calidad de suelos mediante el uso de indicadores e índices. In XVIII Congreso Argentino de la Ciencia del Suelo. 6 p. (CD-ROM).

Cantú, MP; Becker, A; Bedano, AC; Schiavo, HF. 2007. Evaluación de la calidad de suelos mediante el uso de indicadores e índices en la Pampa Argentina. Ci. Suelo (Argentina) 25(2):173-178.

Cantú, MP; Becker, AR; Bedano, JC; Schiviano, HF; Parra, BJ. 2009. Evaluation of the impact of land use and management change by means of soil quality indicators, Cordoba, Argentina. Cadernos Lab. Xeoloxico de Laxe. Coruna 34:203-214.

AGRONOMÍA MESOAMERICANA 24(1):83-91. 2013 
Certini, G; Corti, G; Fernández, S. 2002. Comparison of two soil organic matter extractants and determination of the «Walkley-Black» correction factors for organic fractions from a volcanic soil. Commun. Soil Sci. Plant Anal. 33:685-693.

De la Rosa, DA; Teutli, MM; Ramírez, ME. 2007. Electroremediación de suelos contaminados, una revisión técnica para su aplicación en campo. Rev. Int. Contam. Ambient 23(3):129-138.

Doran, JW; Parkin, TB. 1994. Defining and assessing soil quality. In Doran, JW; Coleman, DC; Bezdicek, DF; Stewart, BA. eds. Defining soil quality for a sustainable environment. SSSA Special Publication $\mathrm{N}^{\circ} 35$. Wisconsin, USA.

Doran, JW; Parkin, TB. 1996. Quantitative indicators of soil quality: a minimum data set. In Methods for assessing Soil quality. SSSA Special Publication $\mathrm{N}^{\circ} 49$. Wisconsin, USA. p. 25-37.

Doran, JW; Sarrantonio, M; Liebig, MA. 1996. Soil health and sustainability. In LD Sparks. ed. Advances in Agronomy, Vol 56. Academic Press Inc. San Diego CA. p. 1-54.

Doran, JW; Safley, M. 1997. Defining and assessing soil health and sustainable productivity. In Pankhurst, C; Doube, BM; Gupta, VVSR. eds. Biological indicators of soil health. CAB International, Wallingford. p. $1-28$.

Doran, JW; Zeiss, MR. 2000. Soil health and sustainability: managing the biotic component of soil quality. Appl. Soil Ecol. 15:3-11.

Gallopin, G. 1997. Indicators and their use: information for decision making. Part 1 Introduction. In Moldan, B; Billharz, S. eds. Sustainability indicators. Wiley, Chichester-N. York, USA. p. 1-13.

Gómez, R; Magallanes, A. 2004. Impacto económico y potencial del uso de bajas densidades de siembra de cebada maltera de temporal en el altiplano Hidalguense. Centro de Investigación General. INIFAP. Folleto técnico $\mathrm{N}^{\circ}$ 3. p. 1-24.

Gregorich, EG; Carter, MR; Angers, DA; Monreal, CM; Ellert, MH. 1994. Towards a minimum data set to assess soil organic matter quality in agricultural soil. Can. J. Soil Sci. 74:367-385

Hati, KM; Swarup, A; Dwivedi, AK; Misra, AK; Bandyopadhyay, KK. 2007. Changes in soil physical properties and organic carbon status at the topsoil horizon of a vertisol of central India after 28 years of continuous cropping, fertilization and manuring. Agriculture, Ecosystems \& Environment 119(1-2):127-134.

Holeplass, H; Singh, BR; Lal, R. 2004. Carbon sequestration in soil aggregates under different crop rotation and nitrogen fertilization in an inceptisol in southeastern Norway. Nutr. Cycl. Agroecosyst 70:167-177.

Jackson, PJ. 1987. Poly ( $\gamma$-glutamylcysteinyl) glycine: Its role in cadmium resistance in plant cells, Proc. Natl. Aca. Sci. 84:6619-6623.

Karlen, DL; Mausbach, MJ; Doran, JW; Cline, RG; Harris, RF; Schuman, GE. 1997. Soil quality: A concept, definition and framework for evaluation. Soil Sci. Soc. Am. J. 61:4-10.

Lado, M; Paz, A; Ben-Hur, M. 2004. Organic matter and aggregate size interactions in infiltration, seal formation and soil loss. Soil Sci. Soc. Am. J. 68:935-942.

Lilburne, I; Saprling, G; Schipper, L. 2004. Soil quality monitoring en New Zealand development of an interpretative framework. Agriculture, Ecosystems \& Environment 104:533-544.

López, P; Guzmán, FA; Santos, EM; Prieto, F; Román, AD. 2005. Evaluación de la calidad física de diferentes variedades de cebada (Hordeum sativum jess) cultivadas en los estados de Hidalgo y Tlaxcala, México. Revista Chilena de Nutrición 32(3):247-253.

Márquez, CO; Garcia, VJ; Cambardella, CA; Schultz, RC; Isenhart, TM. 2004. Aggregate size stability distribution and soil stability. Soil Sci. Soc. Am. J. 68: 725-735.

Medina, J; Volke, VH; González, J; Galvis, A; Santiago, MJ; Cortés, JI. 2006. Cambios en las propiedades físicas del suelo a través del tiempo en los sistemas de maíz bajo temporal y mango bajo riego en luvisoles del estado de Hidalgo. Universidad y Ciencia 22(2): 175-189.

Norma Oficial Mexicana. 2000. NOM-021-RECNAT-2000. Establece las especificaciones de fertilidad, salinidad y clasificación de suelos. Estudios, muestreos y análisis. Diario Oficial de la Federación del 14 de febrero de 2001.p. 17.

OECD (Organisation for Economic Cooperation and Development). 1991. Environmental indicators: A preliminary Set, OCDE, Paris. Personal Laboratorio Salinidad. 1982. Suelos Salinos y Sódicos. Ed. Limusa. Mexico. 172 p.

Prieto, F; Prieto, J; de Ita, S; Méndez, MA; Román, AD. 2009. Correlación de potencial zeta (pZ) y parámetros fisicoquímicos en extractos de saturación de suelos del Distrito de riego 03 del Valle del Mezquital, Hidalgo, 
México. Tropical and Subtropical Agroecosystems 10(2):161-167.

Pulido, MA; Lobo, D; Lozanoi, Z. 2009. Asociación entre indicadores de estabilidad estructural y la materia orgánica en suelos agrícolas de Venezuela. Agrocienc. 43(3):221-230.

Rodríguez, N; Florentino, A; Torres, D; Yendis, H; Zamora, F. 2009. Selection of soil quality indicators in three land use type in the Coro plain. Falcon State. Rev. Fac. Agron. (LUZ) 26:340-361.

Segnestam, L. 2002. Indicators of environmental and sustainable development. Theories and practical experience, environmental economic. Series, Paper $\mathrm{N}^{\circ}$ 89. World Bank, Washington DC. USA. 61 p.

Shepherd, TG; Saggar, S; Newman, RH; Ross, CW; Dando, JL. 2001. Tillage-induced changes to soil structure and organic carbon fraction in New Zealand soils. Aust. J. Soil Res. 39:465-489.

Soil Survey Staff. 2006. Key to soil taxonomy. USDA Tenth Edition. Washington DC. p. 341.

Unzueta, CE; Soto, MA; Martínez, J; Pinto, CF; Cruz, F; Hernández, S. 2007. Electroremediation: a novel technology for the remediation of contaminated soils.
In Memory of Chemical Engineering International Symposium. Durango, México. p. 102-106.

USDA-NRCS (United State Department of Agriculture - Natural Resources Conservation Service). 1999. Soil quality test kit. Section II: Background and interpretive guide for individual tests. Washington DC: Soil Quality Institute. p. 58-83.

Vepraskas, MJ. 1994. Plant response mechanisms to soil compactation. In Wilkinson, RE. ed. Plant-environment interaction. Dekker Publ. Co., New York. p. 263-287.

Vera, JA; Grageda, OA; Vuelvas, MA; Peña, JJ. 2002. Absorción de nitrógeno por el cultivo de cebada en relación con la disponibilidad de agua en el bajío, Guanajuato, México. Terra Latinoamericana 20(1):57-64.

Volveré, B; Amézquita, E. 2009. Estabilidad estructural del suelo bajo diferentes sistemas y tiempo de uso en laderas andinas de Nariño, Colombia. Acta Agron. (Palmira) 58(1):35-39.

Warkentin, BP. 1996. Overview of soil quality indicators. In Cohen, GM; Vanderpluym, HS. eds. Proc. Soil Quality Assessment for the Prairies, Agric. Edmonton, Canadá. p. 1-13. 
